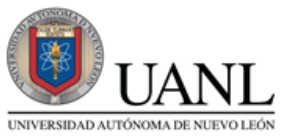

FACPYA
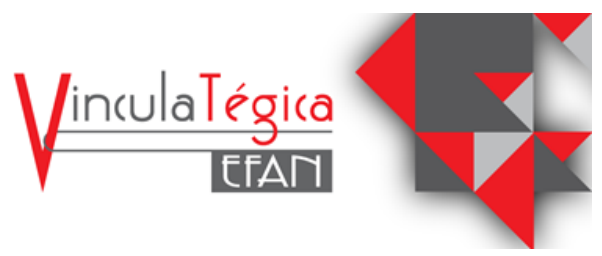

\title{
Efecto de la función psicológica en el bienestar subjetivo de los emprendedores mexicanos
}

\author{
Anais Estefanía González Peña ${ }^{1}$, Yesenia Sánchez Tovar $^{2}$ y César Iván Mellado Ibarra ${ }^{3}$ \\ ${ }^{1}$ Universidad Autónoma de Tamaulipas, a2173048004@alumnos.uat.edu.mx, Boulevar Adolfo López Mateos \\ SN, Centro Universitario, 87149, 8341064631 \\ ${ }^{2}$ Universidad Autónoma de Tamaulipas, yesanchez@docentes.uat.edu.mx, Boulevar Adolfo López Mateos \\ SN, Centro Universitario, 87149, 8342666831 \\ ${ }^{3}$ Universidad Autónoma de Tamaulipas, a2113080034@alumnos.uat.edu.mx, Boulevar Adolfo López Mateos \\ SN, Centro Universitario, 87149, 8343040236
}

Información del artículo revisado por pares

Fecha de aceptación: junio-2021

Fecha de publicación en línea: diciembre-2021

DOI: https://doi.org/10.29105/vtga7.1-106

Resumen

Hoy en día la literatura sobre el emprendimiento ha transitado hacia los resultados que provoca no sólo a nivel económico en el individuo y su territorio, sino también en el bienestar de los emprendedores. El objetivo de este artículo es determinar el impacto de la percepción del funcionamiento personal y social en el bienestar subjetivo de los emprendedores mexicanos. Para la consecución de dicho propósito se plantea una investigación cuantitativa utilizando los datos proporcionados por la encuesta de Bienestar de INEGI (2020). La población estudiada está conformada por emprendedores mayores de edad en México alcanzando una muestra representativa de 480 emprendedores. A través de un análisis factorial y una regresión lineal múltiple se pudo contrastar que el funcionamiento personal y el funcionamiento social de los emprendedores afecta el bienestar subjetivo percibido, lo que permite un aporte a la literatura de la creación de empresas con la finalidad de promover el desarrollo económico del país.

Palabras clave: Bienestar subjetivo, funcionamiento personal, funcionamiento social
Today, the literature on entrepreneurship has moved towards the results it causes not only at the economic level in the individual and his territory, but also in the well-being of entrepreneurs. The objective of this article is to determine the impact of the perception of personal and social functioning on the subjective wellbeing of Mexican entrepreneurs. To achieve this purpose, quantitative research is proposed using the data provided by the INEGI Welfare Survey (2020). The population studied is made up of adult entrepreneurs in Mexico, reaching a representative sample of 480 entrepreneurs. Through a factor analysis and a multiple linear regression it was possible to verify that the personal functioning and social functioning of entrepreneurs affects perceived subjective well-being, which allows a contribution to the literature of the creation of companies with the aim of promoting the economic development of the country.

Keywords: Subjective well-being, personal functioning, social functioning

JEL Codes: L26; M13; D21. 


\section{INTRODUCCIÓN}

El emprendimiento va más allá del crecimiento económico, ya que no solamente se trata de contribuir a un Producto Interno Bruto (PIB) alto, sino que trae consigo importantes consecuencias para los individuos y su entorno. Hoy en día se desarrollan con fuerza corrientes literarias que se están enfocando en observar medidas más robustas para analizar los efectos del emprendimiento, como lo es el bienestar (Abreu et al., 2019; Nikolaev et al., 2020; Weinberg et al., 2018).

A la par y en materia económica, Stiglitz et al. (2009) desarrollaron una teoría denominada "Revisión de la medición del desempeño económico y el progreso social". Ésta fue creada por orden del ex primer presidente francés, Nicolas Sarkozy, con el objetivo de mostrar las limitaciones del PIB como un indicador de la productividad económica y del progreso social, detectando la necesidad de encontrar mediciones complementarias para de medidas globales que indiquen el desarrollo de los individuos $\mathrm{y}$ sus naciones. Como respuesta a esta necesidad, se diseñó el Índice de Bienestar.

Ligando lo anterior al estudio del emprendimiento, algunas investigaciones han destacado que las características individuales y sociales juegan un papel fundamental para el éxito del emprendedor (Chakhar et al., 2020; Garcia-Ramos et al., 2010; Pastora Amador-Ruiz \& Juan Briones-Penalver, 2017) y como se mencionó anteriormente consideran que dichos aspectos asociados a la persona, infieren en los resultados económicos del negocio (Sherman et al., 2016; Su et al., 2020). Sin embargo, en los últimos años ha tomado fuerza la corriente que asocia al emprendimiento con la generación de bienestar (Farny et al., 2019; Hahn et al., 2012, 2012; Lyubomirsky et al., 2005; Nikolaev et al., 2020; Stephan et al., 2020; Wach et al., 2018; Wu et al., 2020).

Pero, ¿qué es el bienestar?, por bienestar se entiende el estado de la persona cuyas condiciones físicas y mentales le proporcionan un sentimiento de satisfacción y tranquilidad (Chadwick \& Raver, 2020;
Farny et al., 2019; Hahn et al., 2012, 2012; Nikolaev, Boudreaux, et al., 2020; Stephan et al., 2020; Wach et al., 2018; Wu et al., 2020).

En relación a lo anterior, el bienestar se encuentra relacionado con una inmensa cantidad de resultados positivos, ya sean estos en el ámbito personal y/o profesional, ya que coadyuva al esfuerzo personal, la satisfacción de ambiciones y la habilidad de lidiar con el estrés (Bockorny \& YoussefMorgan, 2019).

Además, de acuerdo con los autores Lyubomirsky, King y Diener (2005) el bienestar precede a escenarios significativos que se pueden representar a través de un excelente desempeño profesional, relaciones de calidad, incremento de los ingresos y una vida más longeva, tomando el nombre de bienestar subjetivo.

No obstante, el camino para llegar al bienestar no ha sido aún definido totalmente (Nikolaev, 2020), algunos autores apuntan que el bienestar subjetivo es resultado de una percepción psicológica interna del individuo y su filosofía de vida (Voukelatou et al., 2021; Weinberg et al., 2018), mientras que otros autores señalan que el bienestar depende de la percepción del entorno que rodea al individuo (Binder \& Freytag, 2013; Su et al., 2020).

Partiendo de lo antes planteado, la presente investigación tiene por objetivo analizar los determinantes del bienestar subjetivo de los emprendedores. Para la consecución de dicho objetivo se presenta en la siguiente sección una revisión de la literatura relacionada con el bienestar subjetivo, para posteriormente desarrollar una investigación de enfoque cuantitativo que permita analizar los determinantes del bienestar en una muestra de 480 emprendedores. Finalmente se presenta las conclusiones de este trabajo.

\section{MARCO TEÓRICO}

\subsection{Bienestar subjetivo}

$\mathrm{La}$ literatura ha expuesto que el emprendimiento puede incrementar el nivel del trabajo y la satisfacción con la vida, los cuales son factores que conducen hacia el 
bienestar (Binder \& Freytag, 2013; Global Entrepreneurship Monitor, 2020; Stephan et al., 2020).

A su vez, los investigadores han clasificado al bienestar principalmente en dos áreas, las cuales son el bienestar objetivo y el bienestar subjetivo (Voukelatou et al., 2021). El bienestar objetivo refleja las condiciones de vida y la calidad de vida en términos materiales, sin embargo, medir el bienestar subjetivo engloba mayor complejidad (Seligman, 2011).

El bienestar subjetivo engloba los aspectos positivos de experimentar el vivir, combinando los aspectos cognitivos y afectivos del día a día en general, donde predomina la satisfacción con la vida (Diener et al., 1999; Diener et al., 2002). Además, Alexandrova (2005) establece que el término subjetivo es utilizado debido a que permite a los individuos definir lo que les da placer y lo que es relevante para su felicidad. Por lo que se puede medir a través de tres componentes principales, los cuales son el afecto positivo, el afecto negativo y la satisfacción de la vida (Diener \& Suh, 1997).

\subsection{Determinantes del bienestar subjetivo}

En los últimos años, diversa literatura científica ha expresado que los factores del funcionamiento personal y social pertenecientes a la función psicológica, unen la relación existente entre los emprendedores y el bienestar subjetivo, ya que consideran que éste se alcanza después de que un individuo se compromete en la creación de su propia empresa (Nikolaev et al., 2020; Pidd et al., 2014, p. 201; Thompson \& Prottas, 2006; Warr \& Nielsen, 2018).

Por lo que, aun cuando los diversos estudios que involucran los términos del funcionamiento psicológico personal y social como generadores de bienestar subjetivo, este trabajo busca contribuir a la literatura del emprendimiento y al mismo tiempo generar conocimiento que sirva como base para futuras investigaciones. Para su explicación se toma como base la discusión de Nikolaev et al. (2020) que proponen que el funcionamiento personal y social genera el bienestar subjetivo en el emprendedor.
El funcionamiento personal es un concepto multifacético compuesto por la resiliencia, el significado y propósito, la autonomía y control, el compromiso, la competencia y la autoaceptación (Diener et al., 2010).

Autores como Deci \& Ryan (2000), Ryff (1989) y Seligman (2011) establecen que el funcionamiento psicológico, ya sea interno o externo, como el compromiso con la vida, la adaptación a los cambios y contingencias, los talentos y capacidades para la autorrealización, sirven como guías de acción para que las personas experimenten el bienestar subjetivo.

Por funcionamiento social se entienden las relaciones estables y afectivas que generan compromiso hacia los demás, además de que se manifiesta a través de lazos fuertes de amistad y de involucramiento cívico (Diener et al., 2010).

Tomando como punto de partida investigaciones previas (Diener et al., 2002; Nikolaev et al., 2020; Sherman et al., 2016; Warr \& Nielsen, 2018), esta variable se hizo operativa a través del uso de la Escala de Satisfacción con la Vida ESV (Diener et al., 1985) midiendo el grado en que el creador de empresas considera que los factores personales y sociales afectan a su bienestar subjetivo, generando las siguientes hipótesis y su respectivo modelo de investigación.

A partir de lo antes mencionado se establecen las siguientes hipótesis de investigación:

Hipótesis 1: El funcionamiento personal tiene un efecto positivo $y$ significativo en el bienestar subjetivo de emprendedores

Hipótesis 2: El funcionamiento social tiene un efecto positivo y significativo en el bienestar subjetivo de emprendedores. 
Figura 1. Modelo de investigación

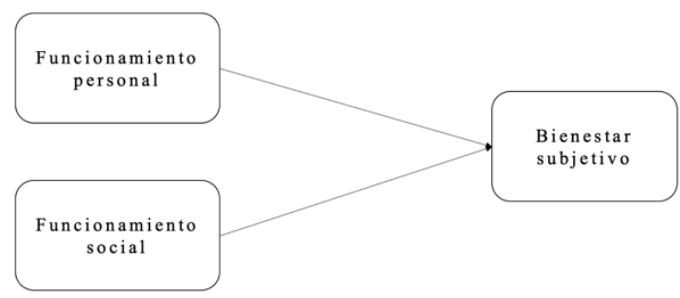

Fuente: Elaboración propia

\section{MÉTODO}

Con el fin de cumplir con el objetivo de investigación propuesto, se diseñó una investigación de corte cuantitativo de tipo causal y transversal. Para lo cual se utilizaron datos de fuentes secundarias obtenidas de la encuesta de Bienestar Autorreportado BIARE Básico diseñado a partir de los trabajos de Stiglitz et al., (2009) y los principios de la OECD de los últimos años (OECD, 2019) aplicado por el INEGI en los meses de enero y octubre de 2020 a ciudadanos mexicanos mayores de 18 años. La base de datos se segmentó a personas que se desempeñaban como dueños de un negocio o autoempleados, logrando así una muestra de 480 individuos considerados como emprendedores. Las técnicas estadísticas utilizadas para el tratamiento de los datos fue el análisis factorial y la regresión lineal múltiple, utilizando el software estadístico SPSS en su versión 24.

Debido a que la hipótesis planteada es referida al funcionamiento personal, el funcionamiento social $y$ el bienestar subjetivo, el estudio de dichos elementos se llevó a cabo a través de las variables que se presentan a continuación:

Tabla 1. Operacionalización

\begin{tabular}{|c|c|c|c|c|}
\hline Variable & Conceptualización & Indicadores utilizados & Escala & Fuente \\
\hline $\begin{array}{l}\text { Funcionamiento } \\
\text { personal }\end{array}$ & $\begin{array}{l}\text { Concepto } \\
\text { multifacético } \\
\text { compuesto por la } \\
\text { resiliencia, } \\
\text { significado el } \\
\text { propósito, } \\
\text { autonomía y control, } \\
\text { el compromiso, la } \\
\text { competencia y la } \\
\text { auto aceptación } \\
\text { (Diener et al., 2010). }\end{array}$ & $\begin{array}{l}\text { - Por lo general siento } \\
\text { que lo que hago en } \\
\text { mi vida vale la pena } \\
\text { - Siento que tengo un } \\
\text { propósito o una } \\
\text { misión en la vida } \\
\text { - Soy libre para } \\
\text { decidir mi propia } \\
\text { vida } \\
\text { - El que me vaya bien } \\
\text { o mal depende } \\
\text { fundamentalmente } \\
\text { de mí } \\
\text { La mayoría de los } \\
\text { días siento que he } \\
\text { logrado algo }\end{array}$ & $\begin{array}{l}\text { Likert } \\
\text { de } 10 \\
\text { puntos }\end{array}$ & $\begin{array}{l}\text { BIARE } \\
\text { básico } \\
2020\end{array}$ \\
\hline
\end{tabular}


- Tengo fortaleza frente a las adversidades

- Soy una persona afortunada

- Siempre soy optimista con respecto a mi futuro

$\begin{array}{ll}\text { Funcionamiento } & \text { Las relaciones } \\ \text { social } & \text { estables y afectivas } \\ & \text { que generan } \\ & \text { compromiso hacia } \\ & \text { los demás, se } \\ & \text { manifiesta a través } \\ & \text { de lazos fuertes de } \\ & \text { amistad, además de } \\ & \text { involucramiento } \\ & \text { cívico (Diener et al., } \\ & \text { 2010). }\end{array}$

\section{Bienestar subjetivo} está con sus

Las respuestas
emocionales de las
personas, el dominio
de los deseos y la
determinación de la
satisfacción con la
vida, por lo que se
recomienda medir
esta variable con la
Escala de
Satisfacción de Vida
de Diener (1985).

- ¿Qué tan satisfecho está con su ciudad?

- ¿Qué tan satisfecho está con su vecindario?

- ¿Qué tan satisfecho relaciones personales?

- ¿Qué tan satisfecho está con su seguridad ciudadana?

- ¿Qué tan satisfecho está con el tiempo del que dispone para hacer lo que le gusta?

- ¿Qué tan satisfecho está con su nivel de vida?

- ¿Qué tan satisfecho está con sus logros en la vida?

- ¿Podría decirme en una escala de 0 a 10 qué tan satisfecho se encuentra actualmente con su vida?

- Y hace un año, ¿qué tan satisfecho se encontraba con su vida?

- En general me siento bien con respecto a mí mismo

\section{Likert BIARE de 10 básico puntos 2020} $\begin{array}{ll}\text { Likert } & \text { Escala de } \\ \text { de 10 } & \text { Satisfacci } \\ \text { puntos } & \text { ón con la } \\ & \text { Vida ESV } \\ & \text { (Diener } \\ & \text { et al., } \\ & 1985)\end{array}$ 


\section{RESULTADOS}

En esta sección se presentan los resultados del análisis de regresión, y la conformación de las variables latentes a través de un análisis factorial exploratorio. En la primera parte, se describen las pruebas de adecuación muestral, la varianza explicada por los factores encontrados, las cargas de cada ítem en las variables latentes, y su fiabilidad medida por el Alfa de Cronbach. En la segunda parte, se explican los resultados del análisis de regresión, las pruebas de bondad de ajuste y el contraste de las hipótesis a través de la significancia estadística de los parámetros estimados.

El análisis factorial se condujo realizando una extracción por máxima verosimilitud, sin una predisposición en el número de elementos que se integrarían en cada variable latente, sin embargo, se contempló que los ítems se agregarían en las tres variables: factores sociales, factores personales y bienestar subjetivo (Cuadras, 1991).

En la Tabla 2, se muestran los resultados del análisis preliminar de los datos para conocer su adecuación para la extracción de los factores. Primero se estimó la medida de adecuación muestral de Kaiser-MeyerOlkin, que tomó el valor de 0.934 , el cual es superior al valor crítico 0.8 por lo que se tiene evidencia de que los datos son adecuados para las estimaciones posteriores; y después la prueba de esfericidad de Bartlett, la cual tuvo un p-valor próximo a 0 , por lo que se concluye que los ítems están intercorrelacionados y son adecuados para el análisis factorial (Lloret-Segura et al., 2014; Martínez \& Sepúlveda, 2012; Romero \& Mora, 2020).

Tabla 2. Resultados de la Medida KMO y Prueba de Bartlett

\begin{tabular}{lll}
\hline Medida de adecuación muestral de Kaiser-Meyer-Olkin. & .934 \\
\hline Prueba de esfericidad de & Chi-cuadrado aproximado & 47500.496 \\
Bartlett & Grados de libertad & 105 \\
& Significancia (P-valor) & .000 \\
\hline
\end{tabular}

Fuente: Elaboración propia.

El siguiente paso en el análisis es la determinación del porcentaje de la varianza que es capturado por los factores en los que se agregan los ítems (Martínez \& Sepúlveda, 2012; Romero \& Mora, 2020). En la Tabla 3 se presenta la cantidad de factores que obtuvieron un eigenvalor mayor que uno, y la proporción de varianza acumulada por cada uno de ellos. Los resultados muestran que los ítems se agregan en tres factores, y explican el $51.99 \%$ de la varianza total.

Tabla 3. Factores con un Eigenvalor mayor a 1 y la Varianza

Explicada.

\begin{tabular}{|c|c|c|c|}
\hline \multirow[t]{2}{*}{ Factor } & \multicolumn{3}{|c|}{ Suma de las saturaciones al cuadrado de la rotación } \\
\hline & Total & $\%$ de la varianza & $\%$ acumulado \\
\hline 1 & 3.696 & 24.638 & 24.638 \\
\hline 2 & 2.466 & 16.443 & 41.081 \\
\hline 3 & 1.637 & 10.915 & 51.996 \\
\hline
\end{tabular}

Fuente: Elaboración propia.

Una vez encontrada la proporción de e la

varianza explicada, se presenta la carga de los ítems y en que factor se agrupan, como 
criterio de selección de los indicadores, se tiene que estos serán adecuados si tienen una carga mayor a 0.5 , sin embargo, el nivel deseable es superior a 0.7 (Lloret-Segura et al., 2014; Medrano \& Muñoz-Navarro, 2017).

En la Tabla 4 se presenta la Matriz de Factores rotados donde se observa, además de la carga factorial, el Alpha de Cronbach que mide la fiabilidad de cada uno de los constructos (Cortina, 1993; Molina et al.,
2013). En dicha tabla se aprecia que el Funcionamiento Personal se compone de 8 indicadores, que tienen una carga superior a 0.5; mientras que en Funcionamiento Social se integran 5 ítems que cumplen con este criterio; por último, Bienestar se construye con 2 elementos con cargas superiores a 0.7. Con respecto al Alfa de Cronbach, se observa que los valores para cada factor son mayores que .7 , lo que indica que existe fiabilidad en los constructos (Escobedo Portillo et al., 2016; Molina et al., 2013).

\section{Tabla 4. Matriz de factores rotados}

\begin{tabular}{|c|c|c|c|c|}
\hline & $\begin{array}{l}\text { Funciona } \\
\text { miento } \\
\text { personal }\end{array}$ & $\begin{array}{l}\text { Funciona } \\
\text { miento } \\
\text { social }\end{array}$ & $\begin{array}{l}\text { Bienestar } \\
\text { subjetivo }\end{array}$ & $\begin{array}{l}\text { Fiabilidad } \\
\text { Alpha de } \\
\text { Cronbach }\end{array}$ \\
\hline La vida vale la pena & .742 & & & 0.890 \\
\hline Propósito o misión en la vida & .707 & & & \\
\hline Libertad de decisión & .683 & & & \\
\hline Aceptación de responsabilidad personal & .644 & & & \\
\hline Logro diario & .588 & & & \\
\hline fortaleza frente a las adversidades & .566 & & & \\
\hline Afortunado con la vida & .552 & & & \\
\hline Optimista con respecto al futuro & .534 & & & \\
\hline Satisfacción con la ciudad & & .655 & & 0.792 \\
\hline Satisfacción con el vecindario & & .625 & & \\
\hline Satisfacción con las relaciones personales & & .538 & & \\
\hline Satisfacción con la seguridad ciudadana & & .530 & & \\
\hline Satisfacción con tiempo libre & & .504 & & \\
\hline Satisfacción actual con la vida & & & .759 & 0.795 \\
\hline Satisfacción años anteriores con la vida & & & .739 & \\
\hline
\end{tabular}


Fuente: Elaboración propia.

Nota: Método de extracción por Máxima verosimilitud. Método de rotación por

Normalización Varimax con Kaiser.

Con los resultados del análisis factorial, se extrajeron los tres factores, y con dichas estimaciones se procedió a realizar un análisis de regresión múltiple por mínimos cuadrados ordinarios, que se describe en la siguiente sección. En la Tabla 5 se presenta un resumen de la estimación de los parámetros de regresión, y los estadísticos de bondad de ajuste del modelo. En primera instancia, se debe determinar si se rechaza la hipótesis nula de que la recta de regresión es significativamente distinta de 0 , para ello se empleó el estadístico F y su p-valor, este tomo un valor de 59.399, que es significativo a un nivel de confianza del .001, por lo que la hipótesis nula es rechazada. Por otro lado, el Coeficiente de Determinación o R2 indica que, aunque es significativamente distinto de 0 la recta de regresión explica solo el $1.7 \%$ de la varianza del constructo Bienestar (Rodríguez-Jaume \& Mora Catalá, 2001). Una vez determinada la bondad de ajuste del modelo, se realizó el contraste de hipótesis con los parámetros de las variables independientes. En la Tabla 5 se aprecia que los parámetros de ambas variables son significativos a un nivel de confianza de 0.001 , y tienen un signo positivo. Por lo tanto, se encontró que el Funcionamiento Personal y el Funcionamiento Social tienen un efecto positivo y significativo en el Bienestar, y las Hipótesis 1 y 2 no se rechazan (Rodríguez-Jaume \& Mora Catalá, 2001).

Tabla 5. Resumen de los resultados de la Regresión Múltiple con Bienestar como variable dependiente.

\begin{tabular}{|c|c|c|c|c|}
\hline \multirow[b]{3}{*}{ (Constante) } & \multicolumn{2}{|c|}{$\begin{array}{l}\text { Coeficientes no } \\
\text { estandarizados }\end{array}$} & \multirow[t]{2}{*}{ Valor $\mathrm{t}$} & \multirow[t]{2}{*}{$\begin{array}{l}\text { Significancia } \\
\text { (P-valor) }\end{array}$} \\
\hline & $\mathrm{B}$ & Desv. Error & & \\
\hline & $-1.27 \mathrm{E}-13$ & ,010 & ,000 & 1,000 \\
\hline Función personal &, 043 &, 012 & 3,651 &, $000 *$ \\
\hline Función social &, 120 &, 013 & 9,341 &, $000 *$ \\
\hline \multirow{3}{*}{$\begin{array}{l}\text { Ajuste del } \\
\text { modelo }\end{array}$} & $\mathrm{F}$ & 59.399 & \multirow[t]{3}{*}{ Sig. <.001* } & \\
\hline & & $\begin{array}{l}.130 \mathrm{a} \\
.017\end{array}$ & & \\
\hline & $\mathrm{R}^{2}$ Corregido & .017 & & \\
\hline
\end{tabular}

Fuente: elaboración propia

Nota: *Significante a un nivel de confianza de 0,001

\section{CONCLUSIONES}

El propósito de esta investigación fue comprobar que los factores personales y los factores sociales impactan de manera positiva en el bienestar subjetivo, donde se identificó que en el sector del emprendimiento, el bienestar subjetivo y satisfacción con la vida está en función de los factores personales y los factores sociales de los creadores de empresas.

Sin embargo, se resalta que la variable función personal y la variable función social, afectan de forma significativa afecta el nivel de bienestar subjetivo de los emprendedores.

Esto apoya los resultados de Nikolaev (2020), los cuales sugieren que el grado de bienestar subjetivo que propician los factores internos y externos del creador de empresas favorecen el desarrollo del 
emprendimiento.

Los resultados deben ser interpretados con cautela considerando que fue un estudio transversal, sin embargo, este análisis tiene un gran valor al aproximar en forma exploratoria la relación entre los factores de personalidad y el bienestar subjetivo.

A su vez los resultados permiten comprobar que en el sector del emprendimiento en México se identifica un alto nivel de bienestar subjetivo debido a que las medias están cercanas al valor 8 en una escala de 1 a 10. Estos resultados son los esperados puesto que el sector de las creación de empresas es considerado relevante en términos de desarrollo económico integral (Stiglitz et al., 2009). El bienestar es el pilar del desarrollo económico de las regiones, en este sentido las organizaciones poco a poco van entendiendo que el funcionamiento personal y social del individuo es un factor positivo para este crecimiento ( $\mathrm{Wu}$ et al., 2020). No obstante, el Estado y las instituciones educativas deben involucrarse generando dinámicas de aprendizaje que fomenten el bienestar en los sectores productivos.

\section{REFERENCIAS}

Abreu, M., Oner, O., Brouwer, A., \& van Leeuwen, E. (2019). Well-being effects of self-employment: A spatial inquiry. Journal of Business Venturing, 34(4), 589-607. https://doi.org/10.1016/j.jbusvent.2018.11.001

Alexandrova, A. (2005). Subjective well-being and Kahneman's 'objective happiness'. Journal of happiness studies, 6(3), 301-324.

Binder, M., \& Freytag, A. (2013). Volunteering, subjective well-being and public policy. Journal of Economic Psychology, 34, 97-119.

Bockorny, K., \& Youssef-Morgan, C. M. (2019). Entrepreneurs' Courage, Psychological Capital, and Life Satisfaction. Frontiers in Psychology, 10, 789. https://doi.org/10.3389/fpsyg.2019.00789

Chakhar, S., Ishizaka, A., Thorpe, A., Cox, J., Nguyen, T., \& Ford, L. (2020). Calculating the relative importance of condition attributes based on the characteristics of decision rules and attribute reducts: Application to crowdfunding. En EUROPEAN JOURNAL OF OPERATIONAL RESEARCH (Vol. 286, Número 2, pp. 689-712). ELSEVIER. https://doi.org/10.1016/j.ejor.2020.03.039

Cortina, J. M. (1993). What is coefficient alpha? An examination of theory and applications. Journal of applied psychology, 78(1), 98.

Deci, E. L., \& Ryan, R. M. (2000). The "what" and "why" of goal pursuits: Human needs and the selfdetermination of behavior. Psychological Inquiry, 11(4), 227-268. https://doi.org/10.1207/S15327965PLI1104_01

Diener, Ed, Suh, E. M., Lucas, R. E., \& Smith, H. L. (1999). Subjective well-being: Three decades of progress. Psychological bulletin, 125(2), 276.

Diener, Ed, Wirtz, D., Tov, W., Kim-Prieto, C., Choi, D., Oishi, S., \& Biswas-Diener, R. (2010). New wellbeing measures: Short scales to assess flourishing and positive and negative feelings. Social 
indicators research, 97(2), 143-156.

Diener, Edward, Lucas, R. E., \& Oishi, S. (2002). Subjective well-being: The science of happiness and life satisfaction. Handbook of positive psychology, 2, 63-73.

Diener, Emmons, R. A., Larsen, R. J., \& Griffin, S. (1985). The satisfaction with life scale. Journal of personality assessment, 49(1), 71-75.

Diener, \& Suh, E. (1997). MEASURING QUALITY OF LIFE: ECONOMIC, SOCIAL, AND SUBJECTIVE INDICATORS. Social Indicators Research, $\quad 40(1), \quad$ 189-216. https://doi.org/10.1023/A:1006859511756

Escobedo Portillo, M. T., Hernández Gómez, J. A., Estebané Ortega, V., \& Martínez Moreno, G. (2016). Modelos de ecuaciones estructurales: Características, fases, construcción, aplicación y resultados. Ciencia \& trabajo, 18(55), 16-22.

Farny, S., Kibler, E., Hai, S., \& Landoni, P. (2019). Volunteer retention in prosocial venturing: The role of emotional connectivity. Entrepreneurship Theory and Practice, 43(6), 1094-1123.

Garcia-Ramos et al. (2010). Características influyentes en el proceso de creación empresarial y en el éxito esperado. Revista Europea de Dirección y Economía de la Empresa, 19, 31-48. https://doi.org/ISSN $1019-6838$

Global Entrepreneurship Monitor. (2020). Global Report 2020 (Núm. 0273649205; pp. 154-154).

Hahn, V. C., Frese, M., Binnewies, C., \& Schmitt, A. (2012). Happy and Proactive? The Role of Hedonic and Eudaimonic Well-Being in Business Owners' Personal Initiative. Entrepreneurship Theory and Practice, 36(1), 97-114. https://doi.org/10.1111/j.1540-6520.2011.00490.x

Lloret-Segura, S., Ferreres-Traver, A., Hernández-Baeza, A., \& Tomás-Marco, I. (2014). El análisis factorial exploratorio de los ítems: Una guía práctica, revisada y actualizada. Anales de Psicología/Annals of Psychology, 30(3), 1151-1169.

Lyubomirsky, S., King, L., \& Diener, E. (2005). The benefits of frequent positive affect: Does happiness lead to success? Psychological bulletin, 131(6), 803.

Martínez, C. M., \& Sepúlveda, M. A. R. (2012). Introducción al análisis factorial exploratorio. Revista Colombiana de Psiquiatría, 41(1), 197-207. https://doi.org/10.1016/S0034-7450(14)60077-9

Medrano, L. A., \& Muñoz-Navarro, R. (2017). Aproximación conceptual y práctica a los modelos de ecuaciones estructurales. Revista digital de investigación en docencia universitaria, 11(1), 219-239.

Molina, J. B., Aranda, L. L., Flores, M. H., \& López, E. J. (2013). Utilización del alfa de Cronbach para validar la confiabilidad de un instrumento de medición de satisfacción del estudiante en el uso del software Minitab MISP. 14-16.

Nikolaev, B., Boudreaux, C. J., \& Wood, M. (2020). Entrepreneurship and Subjective Well-Being: The Mediating Role of Psychological Functioning. Entrepreneurship Theory and Practice, 44(3), 557- 
586. https://doi.org/10.1177/1042258719830314

OECD. (2019). OECD SME and Entrepreneurship Outlook 2019. https://doi.org/10.1787/34907e9c-en

Pastora Amador-Ruiz, J., \& Juan Briones-Penalver, A. (2017). Study of female entrepreneurship: An empirical evidence in the Municipality of Leon in Nicaragua. En TOURISM \& MANAGEMENT

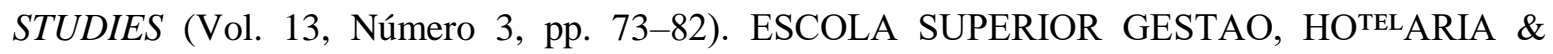
TURISMO UNIV ALGARVE. https://doi.org/10.18089/tms.2017.13308

Pidd, K., Roche, A. M., Fischer, J. A., \& McCarthy, C. (2014). Risky behaviours, risky work settings: The alcohol and drug consumption patterns, health and wellbeing of commercial cookery trainees. Journal of Health, Safety and Environment, 30(2), 301-311.

Rodríguez-Jaume, M.-J., \& Mora Catalá, R. (2001). Análisis de regresión múltiple. Técnicas de investigación social II.

Romero, K. P., \& Mora, O. M. (2020). Análisis factorial exploratorio mediante el uso de las medidas de adecuación muestral kmo y esfericidad de bartlett para determinar factores principales. Journal of Science and Research: Revista Ciencia e Investigación. ISSN 2528-8083, 5(CININGEC), 903-924.

Ryff, C. D. (1989). Beyond Ponce de Leon and Life Satisfaction: New Directions in Quest of Successful Ageing. International Journal of Behavioral Development, 12(1), 35-55. https://doi.org/10.1177/016502548901200102

Seligman, M. (2011). Flourish: A visionary new understanding of happiness and well-being. Policy, 27(3), $60-61$.

Sherman, C. L., Randall, C., \& Kauanui, S. K. (2016). Are you happy yet? Entrepreneurs' subjective wellbeing. Journal of Management, Spirituality \& Religion, 13(1), 7-23. https://doi.org/10.1080/14766086.2015.1043575

Stephan, U., Rauch, A., \& Hatak, I. (2020). The Happy Entrepreneur? A Meta-Analysis of Entrepreneurship and Well-Being Considering Context. 2020(1), 16967.

Stiglitz, J., Sen, A., \& Fitoussi, J.-P. (2009). The Measurement of Economic Performance and Social Progress Revisited (Núm. 33). OFCE - Centre de recherche en économie de Sciences Po.

Su, Y., Zahra, S. A., Li, R., \& Fan, D. (2020). Trust, poverty, and subjective wellbeing among Chinese entrepreneurs. Entrepreneurship \& Regional Development, 32(1-2), 221-245. https://doi.org/10.1080/08985626.2019.1640483

Thompson, C. A., \& Prottas, D. J. (2006). Relationships among organizational family support, job autonomy, perceived control, and employee well-being. Journal of occupational health psychology, 11(1), 100.

Voukelatou, V., Gabrielli, L., Miliou, I., Cresci, S., Sharma, R., Tesconi, M., \& Pappalardo, L. (2021). Measuring objective and subjective well-being: Dimensions and data sources. International Journal of Data Science and Analytics, 11(4), 279-309. https://doi.org/10.1007/s41060-020-00224-2 
Wach, D., Stephan, U., Marjan, J. G., \& Wegge, J. (2018). Entrepreneurs' achieved success: Developing a multi-faceted measure. International Entrepreneurship and Management Journal, 1-29.

Warr, P., \& Nielsen, K. (2018). Wellbeing and work performance. Handbook of well-being. Salt Lake City, UT: DEF Publishers.

Weinberg, M. K., Seton, C., \& Cameron, N. (2018). The Measurement of Subjective Wellbeing: Item-Order Effects in the Personal Wellbeing Index-Adult. Journal of Happiness Studies, 19(1), 315-332. https://doi.org/10.1007/s10902-016-9822-1

Wu, A., Song, D., \& Yang, Y. (2020). Untangling the effects of entrepreneurial opportunity on the performance of peasant entrepreneurship: The moderating roles of entrepreneurial effort and regional poverty level. Entrepreneurship \& Regional Development, 32(1-2), 112-133. https://doi.org/10.1080/08985626.2019.1640479 